ANNALES

POLONICI MATHEMATICI

XLVI (1985)

\title{
On some classes of infinitely decomposable Sobolev-Schwartz test functions
}

\author{
by JAN Mikusı́nski (Katowice)
}

Franciszek Leja in memoriam

\begin{abstract}
There are considered three different subspaces of Schwartz's space $\mathscr{D}$ such that each element splits to a convolution of two elements of the same subspace.

We are going to define some subclasses $\mathscr{D}_{0}$ of $\mathscr{D}$ such that each function $\varphi \in \mathscr{D}$ is decomposable in $\mathscr{D}_{0}$, i.e. $\varphi=\varphi_{1} * \varphi_{2}$, where $\varphi_{1} \in \mathscr{D}_{0}$ and $\varphi_{2} \in \mathscr{D}_{0}$. We shall first construct the Fourier transforms of functions belonging to $\mathscr{D}_{0}$.

Let $G(z)(z=x+i y)$ be a function of exponential type, not identically 0 , such that
\end{abstract}

$$
\begin{gathered}
|G(z)| \leqslant e^{\alpha|y|}, \\
|1-G(z)| \leqslant A|z| \quad \text { for }|z|<1, \\
|G(t)| \leqslant B /|t| \quad \text { for } t \leqslant-1 \text { and } t \geqslant 1,
\end{gathered}
$$

where $A, B$ and $\alpha$ are positive constants.

THEOREM. If $\lambda_{n} \in \boldsymbol{R}$ and $\sum_{n=1}^{\infty}\left|\lambda_{n}\right|<\infty$, then the product

$$
F(z)=\prod_{n=1}^{\infty} G\left(\lambda_{n} z\right)
$$

represents an entire function, not identically 0 such that

$$
|F(z)| \leqslant e^{\beta|y|}
$$

and

$$
\int_{-\infty}^{\infty}\left|t^{k} F(t)\right| d t \leqslant M_{k}
$$

where $\beta$ and $M_{k}$ are positive constants and $k=1,2, \ldots$

13 - Annales Polonici Mathematici XLVI 
Proof. By (2), we have

$$
\left|1-G\left(\lambda_{n} z\right)\right| \leqslant A\left|\lambda_{n}\right| \cdot|z| \quad \text { for }|z|<1 /\left|\lambda_{n}\right| .
$$

Since $1 /\left|\lambda_{n}\right| \rightarrow \infty$, the series

$$
\sum_{n=1}^{\infty}\left|1-G\left(\lambda_{n} z\right)\right|
$$

is uniformly convergent on compact sets and thus the product in (4) represents an entire function which is not identically 0 .

By (1), we obtain

$$
\left|G\left(\lambda_{n} z\right)\right| \leqslant \exp (\beta|y|)
$$

where $\beta \in \alpha\left(\sum_{n=1}^{\infty}\left|\lambda_{n}\right|\right)$, so (5) holds.

Since, by (1),

$$
|G(t)| \leqslant 1 \quad \text { for real } t
$$

we have

$$
\left|t^{k} F(t)\right| \leqslant\left|t^{k} \prod_{n=1}^{k+2} G\left(\lambda_{n} t\right)\right|
$$

Hence, in view of (3), we get

$$
\left|t^{k} F(t)\right| \leqslant C_{k} / t^{2} \quad \text { for }|t| \geqslant a_{k}
$$

and

$$
\left|t^{k} F(t)\right| \leqslant|t|^{k} \quad \text { for }|t|<a_{k}
$$

where

$$
C_{k}=B^{k+2}\left|\lambda_{1}, \ldots, \lambda_{k+2}\right|^{-1} \quad \text { and } \quad a_{k}=\max \left(\frac{1}{\left|\lambda_{1}\right|}, \ldots, \frac{1}{\left|\lambda_{k+2}\right|}\right) .
$$

Relations (7) and (8) imply (6) and the proof is completed.

As a function $G$ satisfying conditions (1)-(3) we can take $\frac{\sin z}{z}$. Another function of that type is $G(z)=(\exp i z-1) / z$.

By the Paley-Wiener theorem, the function $F(z)$ in (2) is the Fourier transform of the function

$$
f(s)=\frac{1}{2 \pi} \int_{-\infty}^{\infty} e^{-i s t} F(t) d t
$$


whose support is contained in $[-\beta, \beta]$. Moreover, condition (6) guarantees that $f(s) \in C^{x}$ and

$$
f^{(k)}(s)=\frac{1}{2 \pi} \int_{-\infty}^{\infty}(i t)^{k} e^{-i s t} F(t) d t .
$$

Therefore to each function $G$ satisfying (1) $(3)$ there corresponds a class $\mathscr{D}_{0}=\mathscr{D}_{G} \subset \mathscr{D}$ of functions, depending on the sequence $\lambda_{n}$, whose Fourier transforms are equal $F$.

Evidently, if we split the sequence $\lambda_{n}$ in two subsequences $\lambda_{n}^{\prime}$ and $\lambda_{n}^{\prime \prime}$, then $\sum_{n=1}^{\infty}\left|\lambda_{n}^{\prime}\right|<\infty$ and $\sum_{n=1}^{\infty}\left|\lambda_{n}^{\prime \prime}\right|<\infty$ and

$$
F(z)=F_{1}(z) \cdot F_{2}(z)
$$

where

$$
F_{1}(z)=\prod_{n=1}^{\infty} G\left(\lambda_{n}^{\prime} z\right) \quad \text { and } \quad F_{2}(z)=\prod_{n=1}^{\infty} G\left(\lambda_{n}^{\prime \prime} z\right) .
$$

That means, we have $\varphi=\varphi_{1} * \varphi_{2}$, where $\varphi, \varphi_{1}, \varphi_{2}$ are the functions of the class $\mathscr{D}_{0}$, corresponding to the functions $F, F_{1}, F_{2}$, respectively. Thus we have

$$
\mathscr{D}_{0}=\mathscr{D}_{0} * \mathscr{D}_{0} \text {. }
$$

Another class $\mathscr{D}_{0}$ with this property was given in the paper: L. A. Rubel, W. A. Squires and B. A. Taylor, Irreducibility of certain entire functions with applications to harmonic functions, Ann. of Math. 108 (1976), $560-561$. The method of the authors consisted in considering zeros of the Fourier transforms of $\varphi \in \mathscr{Z}$.

Piotr Mikusiński has remarked that a class $\mathscr{D}_{0} \in \mathscr{D}$ with property (9) can be easily constructed directly, without any help of Fourier transform. In fact, there are functions $\delta_{n} \in \mathscr{D}$ such that $\delta_{n} \geqslant 0, \int \delta_{n}=1$ and the support of $\delta_{n}$ is in $\left[-\lambda_{n}, \lambda_{n}\right]$, where $\sum_{n=1}^{\infty} \lambda_{n}<\infty$. We put $\varphi \in \mathscr{O}_{0}$ if $\varphi=\delta_{1} * \delta_{2} * \ldots$ Then and (9) holds, because we may set $\varphi_{1}=\delta_{1} * \delta_{3} * \ldots, \varphi_{2}=\delta_{2} * \delta_{4} * \ldots$

The class of Piotr Mikusiński is the simplest, but does not include the preceding classes. The variety of possible subclasses satisfying (9) suggests that we possibly have $\mathscr{D}=\mathscr{D} * \mathscr{D}$ for the whole class $\mathscr{D}$. 